\title{
The computer training program on tomography
}

\author{
A. Bulba, L. Luizova, A. Khakhaev
}

A. V. Bulba, L. A. Luizova, A. D. Khakhaev, "The computer training program on tomography," Proc. SPIE 9663, Eighth International Topical Meeting on Education and Training in Optics and Photonics, 96632G (6 October 2003); doi: $10.1117 / 12.2207468$

SPIE Event: Eighth International Topical Meeting on Education and Training in Optics and Photonics, 2003, Tucson, Arizona, United States 


\title{
The computer training program on tomography
}

\author{
A.V. Bulba, L.A. Luizova, A.D. Khakhaev \\ Petrozavodsk State University, 33 Lenin St., Petrozavodsk, 185640, Russia \\ alim@karelia.ru
}

\begin{abstract}
Computer training program was developed to learn the students, studying plasma diagnostics methods, the principles of computer tomography and some algorithms of reconstruction of 3D plasma parameter distributions. The program allows to simulate some spatial distribution of plasma emission, to calculate projections of non-uniform plasma objects and to demonstrate visually the result of restoration of axially symmetric and asymmetric objects at the chosen number of projections. The software allows to simulate the noise distortion of projections and thus to show the dependence of reconstruction accuracy on the number of projections, noise level and reconstruction algorithm.

Key words: training program, tomography, plasma, help system, projection, iterative methods, analytical methods, computer simuting, filtration by convolution.

(C)2003 Optical Society of America

OCIS codes: (000.2060) Education, (110.6960) Tomography
\end{abstract}

\section{Introduction}

One of the modern methods of plasma diagnostics is the method of the reconstructive computer tomography. This method allows determining the local characteristics of researched heterogeneous plasma objects with an enough high degree of accuracy that gives broad opportunities in understanding physical processes in these objects. The quality of the reconstruction essentially depends on such factors, as the number of directions of observation, the number of detecting devices, the stability of the algorithm to noises.

The computer simulation allows determining the optimal conditions of the statement of the tomography experiment.

In this connection there was a task to create the training software, which would allow acquainting a student with some algorithms of the computing tomography and with the conditions of the realization of the tomography experiment.

The developed training program «Tomography $1 »$ allows:

1. to calculate projections of mathematical models of heterogeneous plasma objects,

2. to visually demonstrate the reconstruction of axially symmetric and asymmetric objects at the chosen number of projections (perspectives of supervision),

3. to add noise on projections,

4. to represent the results of reconstruction in a three-dimensional form and to have the possibility to normalize it,

5. to determine the accuracy of restoration,

6. to analyse the dependence of the accuracy of restoration of an object on the number of projections and noise level.

Our software contains three algorithms of computer tomography: "method of reverse projections", "wavelet filtration" [1] and "the filtration by convolution" based on Fourier transform [1].

In spite of the fact that the method of reverse projection restores the image with significant false signals and now is used in rare cases, it is cited here due to its importance among the basic precise methods.

The method of reverse projections with the filtration is used in many modern X-ray diagnostic tomographs and thus plays the special role from the practical point of view.

Also in the software two iterative methods of tomography are realized: an algebraic method of restoring (ARTAlgebraic Reconstruction Technigues) or a beam by beam correction and iterative method of least squares (ILST Iterative Least-Squares Technique) or simultaneous correction [2]. 
The demonstrating the work of both analytical and iterative methods gives more ideas in computing tomography since they were widely introduced in various applications and played the important role in development of tomography.

The program was developed in Borland Delphy 7.0. "Tomography 1" requires operational system Microsoft Windows 95/98/2000/XP on IBM PC the AT-compatible computer with the processor above Celeron 266. At least, work of the program needs 1 Mbyte of free disk memory (6 Mbytes including help instructions). The videodriver must be set up in High Color or True Color mode. The program uses standard library OpenGL.

\section{Tomography application to plasma diagnostics}

For determination of local plasma parameters - electron, ion and atom densities and energy distribution functions such optical characteristics of elementary plasma volume as the refraction index $n(\lambda, r)$, the absorption coefficient $k(\lambda, \mathrm{r})$, the spectral emissivity $\varepsilon(\lambda, \mathrm{r})$ can be used. Here $\lambda$ - wavelength, $r$ - coordinate of some point in plasma volume.

But these values are not measurable in inhomogeneous plasma. Only following parameters of a source can be measured:

$$
\begin{aligned}
& b(\lambda, r) \text { - spectral surface radiance, } Q(\lambda, r) \text { - phase difference, } \tau(\lambda, r) \text { - optical thickness of plasma. } \\
& \mathrm{b}(\lambda, \mathrm{v})=\int \varepsilon(\lambda, \mathrm{u}) \mathrm{du}, \theta(\lambda, \mathrm{v})=\int \mathrm{n}(\lambda, \mathrm{u}) \mathrm{du}, \tau(\lambda, \mathrm{v})=\int \mathrm{k}(\lambda, \mathrm{u}) \mathrm{du}
\end{aligned}
$$

The integration in all cases is conducted along "of a ray of observation" $U$, and scanning is possible along a perpendicular direction $\mathrm{V}$, i.e. all listed integrated values can be considered as a projection and to restore local characteristics $n(\lambda, \mathrm{r}), k(\lambda, \mathrm{r}), \varepsilon(\lambda, \mathrm{r})$ one have to use tomography methods.

\section{The description of the program and its applicatoin}

At the start of the program "Tomography 1.exe" there is a main window on a screen - "Tomography 1" (Fig. 8.), on

\begin{tabular}{|c|c|}
\hline 圈Tomograph & \\
\hline File Options & He-lp \\
\hline 目 Save graph & $\mathrm{Ctrl}+\mathrm{S}$ \\
\hline II Exit & $C t r l+X$ \\
\hline
\end{tabular}
which the components to set parameters and to control the work of the program are located:

Fig. 2.1. The «File» menu.

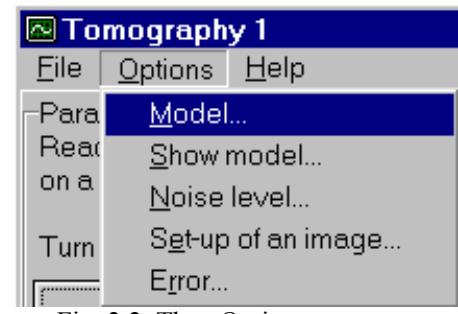

Fig. 2.2. The «Options» menu.

\section{圆Tomography 1}

File Options Help

Parameters of \& Iomography1 Help

Fig. 2.3. The «Help» menu.
The «File» menu (Fig. 2.1.) of the main window allows to quite the program (Exit) and to save of the obtained pictures in BMP a format (Save graph).

From the «Options» menu (Fig. 2.2.) the modules for the determination of a mathematical model (Model ...), for the presentation of the given model in three dimension kind (Show model ...), for determination of noise level (Noise level ...), for parameter setup of restored object (Set-up of an image ...) and for information about accuracy of object's restoring (Error ...) are called.

From the «Help» menu (Fig. 2.3) the in-depth help of the program's description "Tomography 1 " and methodical information on tomography (concept of tomography, mathematical description of tomography methods, features of plasma objects tomography etc.) are called.

For deriving submission about conditions of tomography experment realization and in-depth inspection with some algorithms of computing tomography, first of all it is necessary for student to study closely a reference system called from the program (Fig. 2.3.).

The file of help represents itself some chapters with methodical information on tomography, and also contains the description of the program (Fig. 2.4.). 


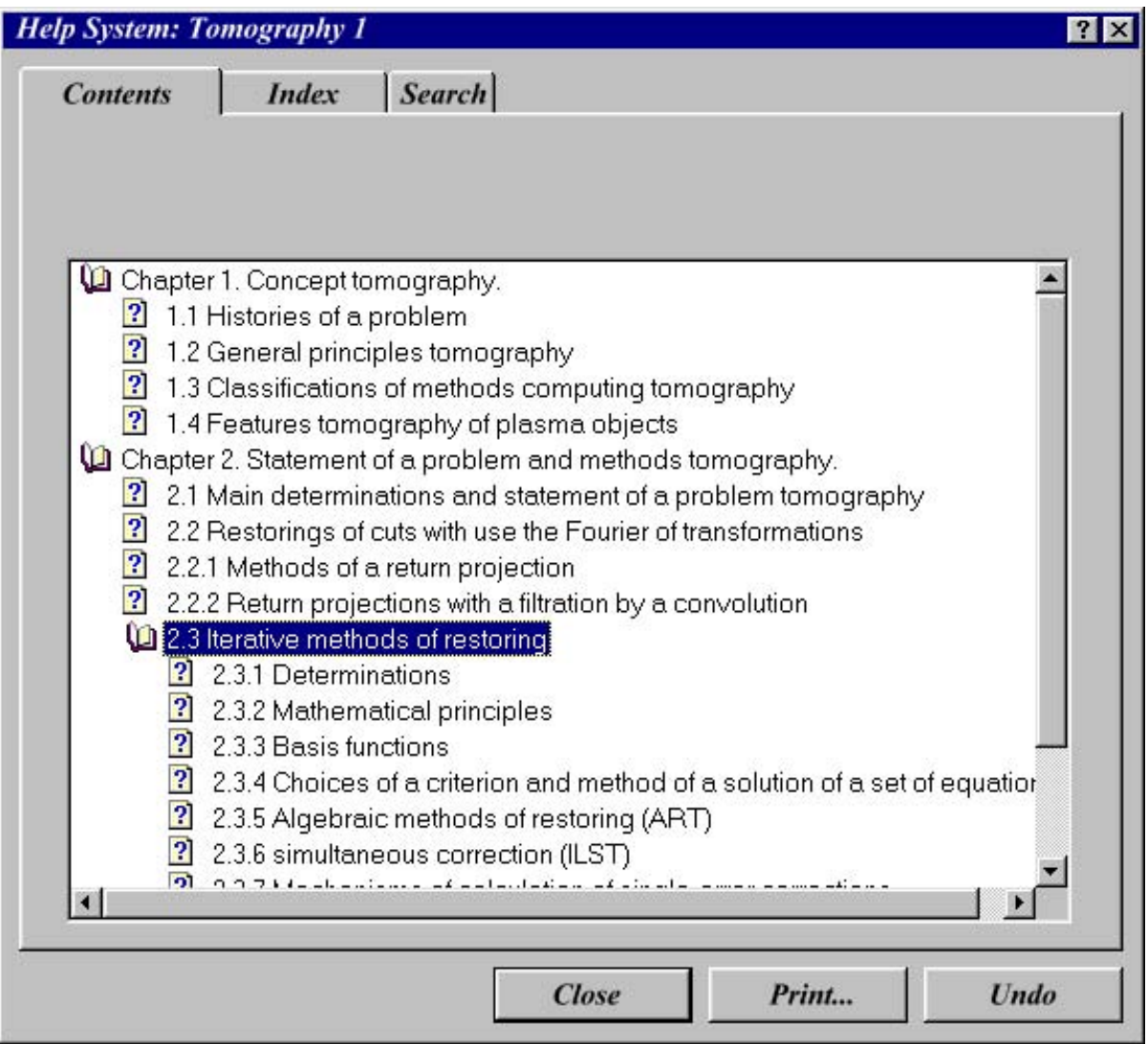

Fig. 2.4. A reference system of the program Tomography 1.

From a reference system student learns, for example, that the methods computing tomography can be divided into two main classes: analytical and iterative [2,3].

The analytical methods are based on point mathematical solutions of the image reconstruction equations. A Fourier transform and Radon's transform are used in a basis of majority of them. All analytical methods of image reconstruction theoretically are equivalent; however, they differ by implementing procedure.

The iterative methods of image reconstruction use approximation of restored object by an array of cells of an equal denseness representing themselves unknown quantities connected by a system of the linear algebraic equations, free terms of which are the readout on a projection. The set of equations are decided by iterative methods and it has denominated the given class of restoring methods. Some iterative methods of image reconstruction now are known. They differ from each other on a sequence of entering of single-error corrections during iteration. Among them three methods are most known and common: an algebraic method of restoring (ART), method of simultaneous iterative restoring (SIRT) and iterative method of least squares (ILST)[2].

After an inspection of a theoretical part, student is offered to begin immediately realization tomographic experiment on the basis of numerical simulating. 


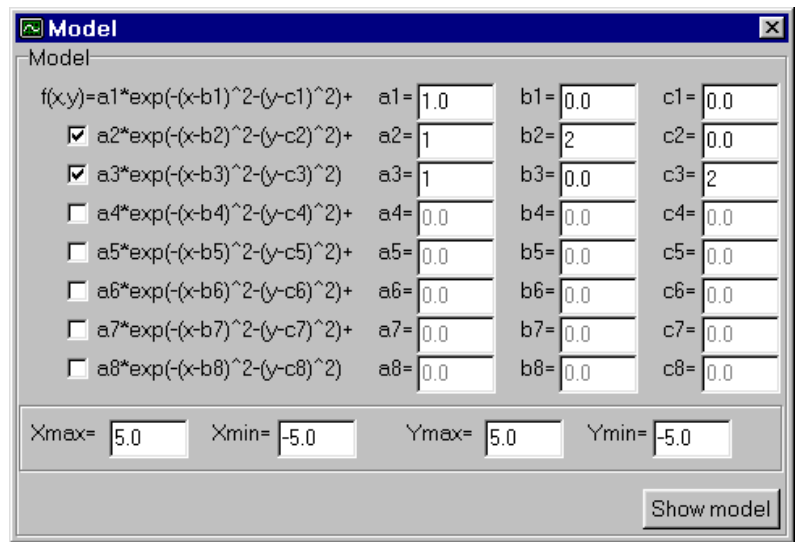

Fig. 2.5. Modelling of a mathematical model.
For this purpose from the «Options» menu of a main window, the module for the task of a mathematical model (Model ...) (Fig. 2.5) is called. Student is offered to use various combinations a Gaussian distribution in quality of enough smooth model distributions simulating spatially an inhomogeneous medium. In the window user selects a combination of functions of the Gauss (up to 9 pieces),

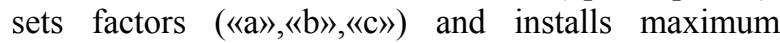
(Xmax, Ymax) and minimum (Xmin, Ymin) significances on axeses. The button «Show model» is pressed further for show created model in the separate window «Model» (Fig. 2.6.).

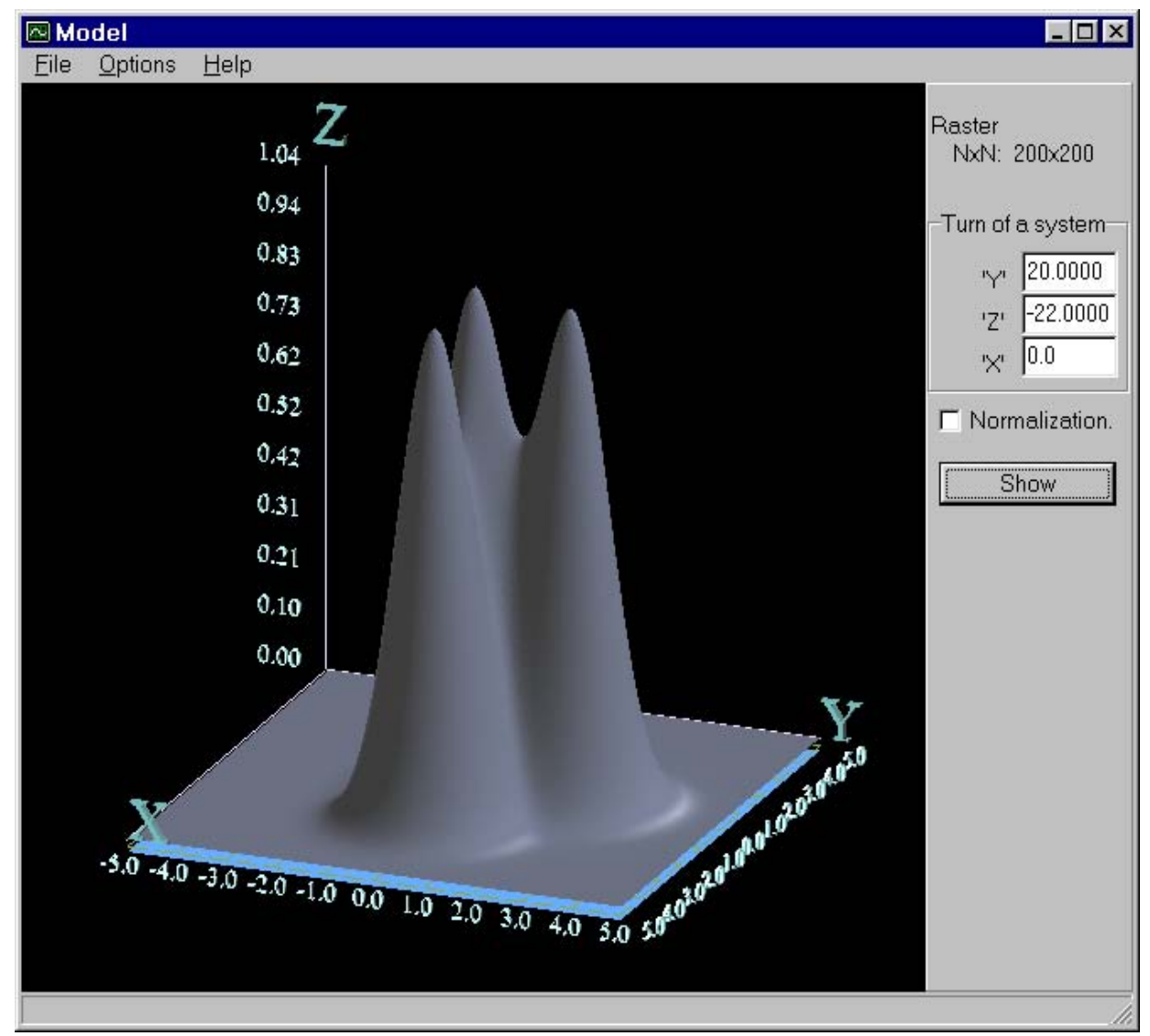

Fig. 2.6. The model created by the user.

In a right upper angle of the «Model» window (Fig. 2.6.) the raster of a model (Raster NxN) is underlined, «Turn of a system» - shows turn angles of a frame («X», «Y»), «Z»). Simple movement of the Mouse stipulates the possibility of a turn of a model. The button «Show» should be pressed in case of redrawing the picture. Normalization is selected, if it is necessary to realize a normalization of a model (at determination of an error of restoring normalization happens automatically). There are the same items in the menu « File», «Options» «Help» as in the main window.

After the model is given, it is necessary to return to the main window (Fig. 2.9.) (It is open all the time). The components located on it allow conducting numerical experiment.

Student is offered to look through projections from the created mathematical model. In 


\begin{tabular}{|l}
$\begin{array}{l}\text { Parameters of a projection } \\
\text { Samples } \\
\text { on a projection: } \\
\text { Turn angle: }\end{array}$ \\
\hline Projection \\
\hline Nucleus \\
\hline Nucleus Ram \\
\hline Convolution \\
\hline
\end{tabular}

Fig. 2.7. Parameters of a projection.

the block «Parameters of a projection» (Fig. 2.7.), user sets number of samples - «samples on a projection», the turn angle of a frame «Turn angle» and presses a button «Projection». For a conclusion of nucleus convolution function, the nucleus (Nucleus of Ramachandrana or MHAT (Mexican hat) wavelet ))is selected and the button «Nucleus» is pressed. (Fig. 2.8,9). For survey of a nucleus convolution with the introduced projection "Convolution» is pressed (Fig. 2.10,11). For it student should compare obtained outcomes and on the basis of information from a reference system qualitatively predict exactitude of restoring by various algorithms.

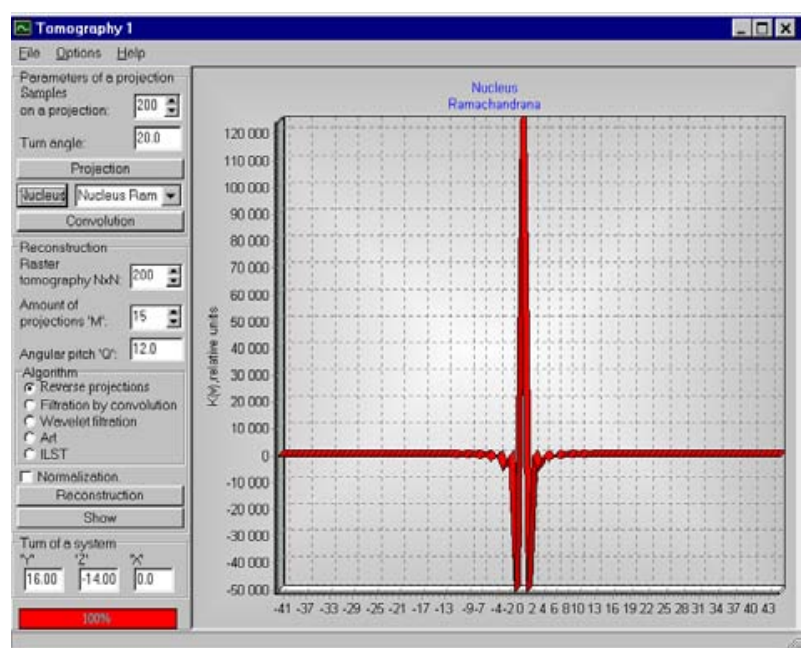

Fig. 2.8. A nucleus Ramachandran.

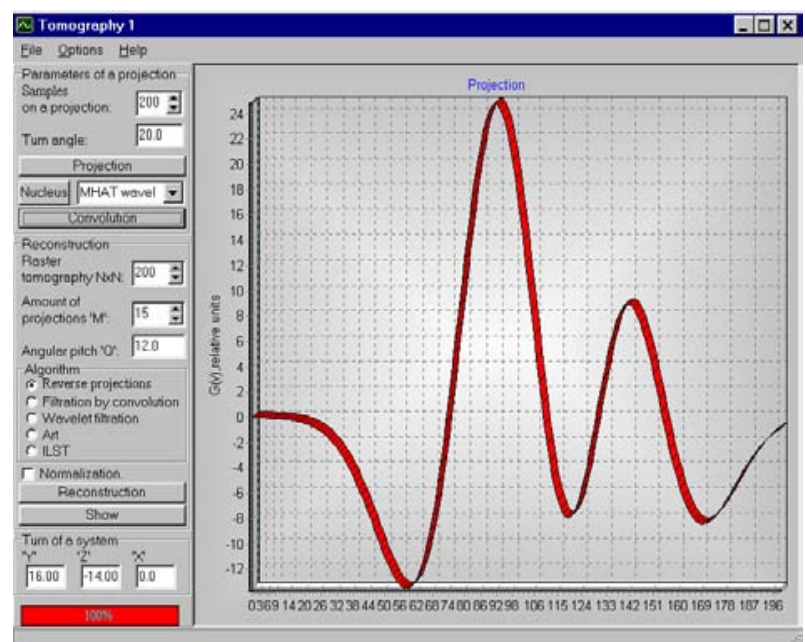

Fig. 2.10. A convolution of a projection with MHAT wavelet.

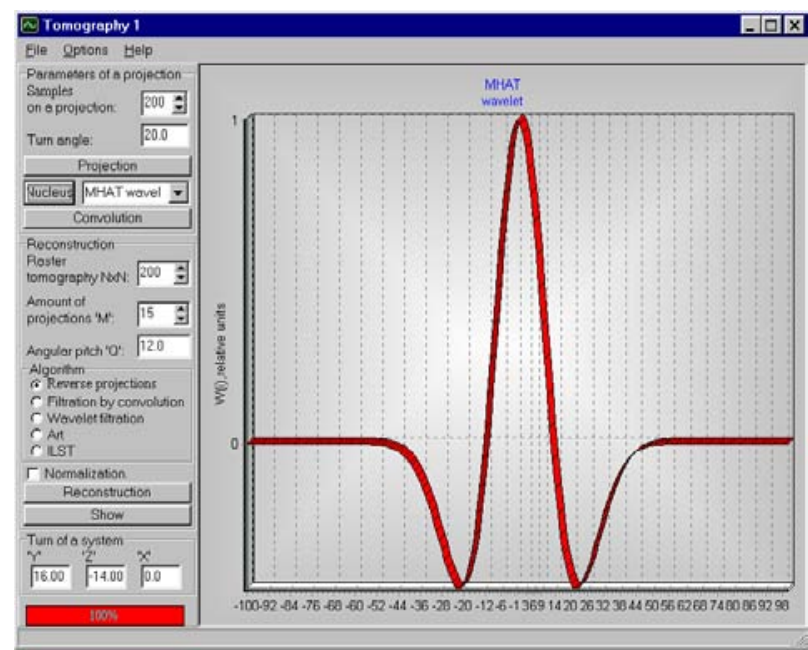

Fig. 2.9. MHAT wavelet.

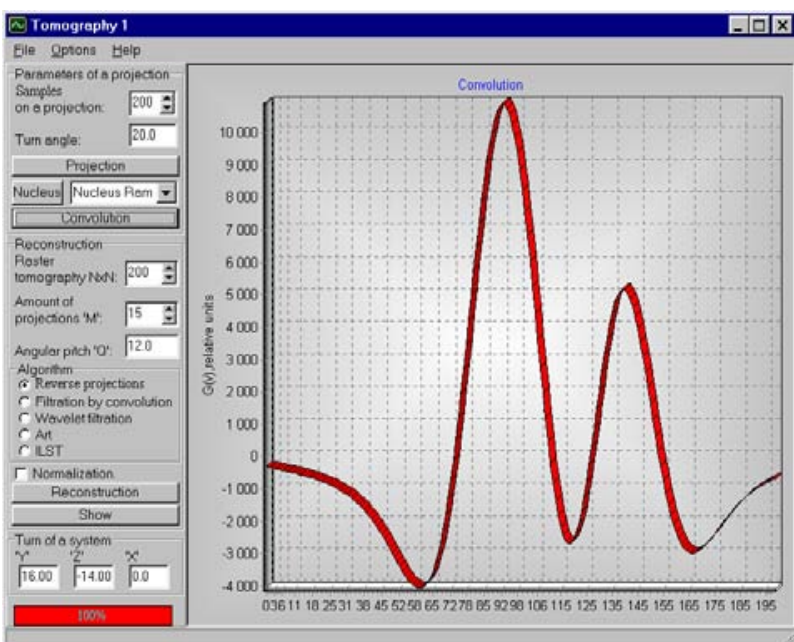

Fig. 2.11. A convolution of a projection with a nucleus Ramachandran. 


\begin{tabular}{|c|c|}
\hline $\begin{array}{l}\text { Reconstruction } \\
\text { Raster } \\
\text { tomography NxN: }\end{array}$ & $200 \div$ \\
\hline $\begin{array}{l}\text { Amount of } \\
\text { projections ' } M \text { ': }\end{array}$ & $15 \div$ \\
\hline Angular pitch 'Q': & 12.0 \\
\hline $\begin{array}{l}\text { Algorithm } \\
\odot \text { Reverse project } \\
\odot \text { Filtration by cor } \\
\odot \text { Wavelet filtratio } \\
\odot \text { Art } \\
\odot \text { ILST }\end{array}$ & ivolution \\
\hline \multicolumn{2}{|l|}{ Г Normalization. } \\
\hline \multicolumn{2}{|c|}{ Reconstruction } \\
\hline Show & \\
\hline
\end{tabular}

Fig. 2.12. Reconstruction.

\begin{tabular}{|c|c|}
\hline Error & $x$ \\
\hline Error & \\
\hline $14.180 \%$ & $\begin{array}{l}\text { Area of errors } \\
\text { The absolute error. }\end{array}$ \\
\hline To calculate & To calculate \\
\hline
\end{tabular}

Fig. 2.13. A conclusion of information about exactitude of restoring.
For direct restoring of a model there are set in the block «Reconstruction» (Fig. 2.12.):

Raster of tomogram «Raster tomography $\mathrm{NxN} »$; number of projections «Amount of projections M»; an angular pitch «Angular pitch»; algorithm of restoring «Algorithm»; it is underlined whether to conduct a normalization «Normamalization» and then the button «Reconstruction» is pressed.

For displaying a restored object the button «Show» is to be pressed.

For best understanding of algoritm operating, student should implement a series of experiments varying parameters of restoring, should make the comparative qualitative analysis.

For realization of the quantitative analysis, it is necessary from the «Options» menu (Error ...) of a main window to call the module (Fig. 2.13.) of information conclusion about accuracy of object reconstruction (Error). Quality measure of reconstruction is an error of restoring magnitude $\Delta(1)$, which is determined as root-mean-square norm of a restored solution deviation $f\left(x_{i}, y_{j}\right)$ from point $f_{0}\left(x_{i}, y_{j}\right)[4]$

$$
\Delta=\frac{\left(\sum_{i} \sum_{j}\left(f\left(x_{i}, y_{j}\right)-f_{0}\left(x_{i}, y_{j}\right)\right)^{2}\right)^{1 / 2}}{\left(\sum_{i} \sum_{j}\left(f_{0}\left(x_{i}, y_{j}\right)\right)^{2}\right)^{1 / 2}} \cdot 100 \%
$$

As the independent task student is offered to conduct a series of experiments and stydy the dependance of reconstruction accuracy on the number of projections and to determine whether in all algorithms the magnification of projections number leads to increasing of restoring exactitude.

Images with restored models by various algorithms are indicated below.

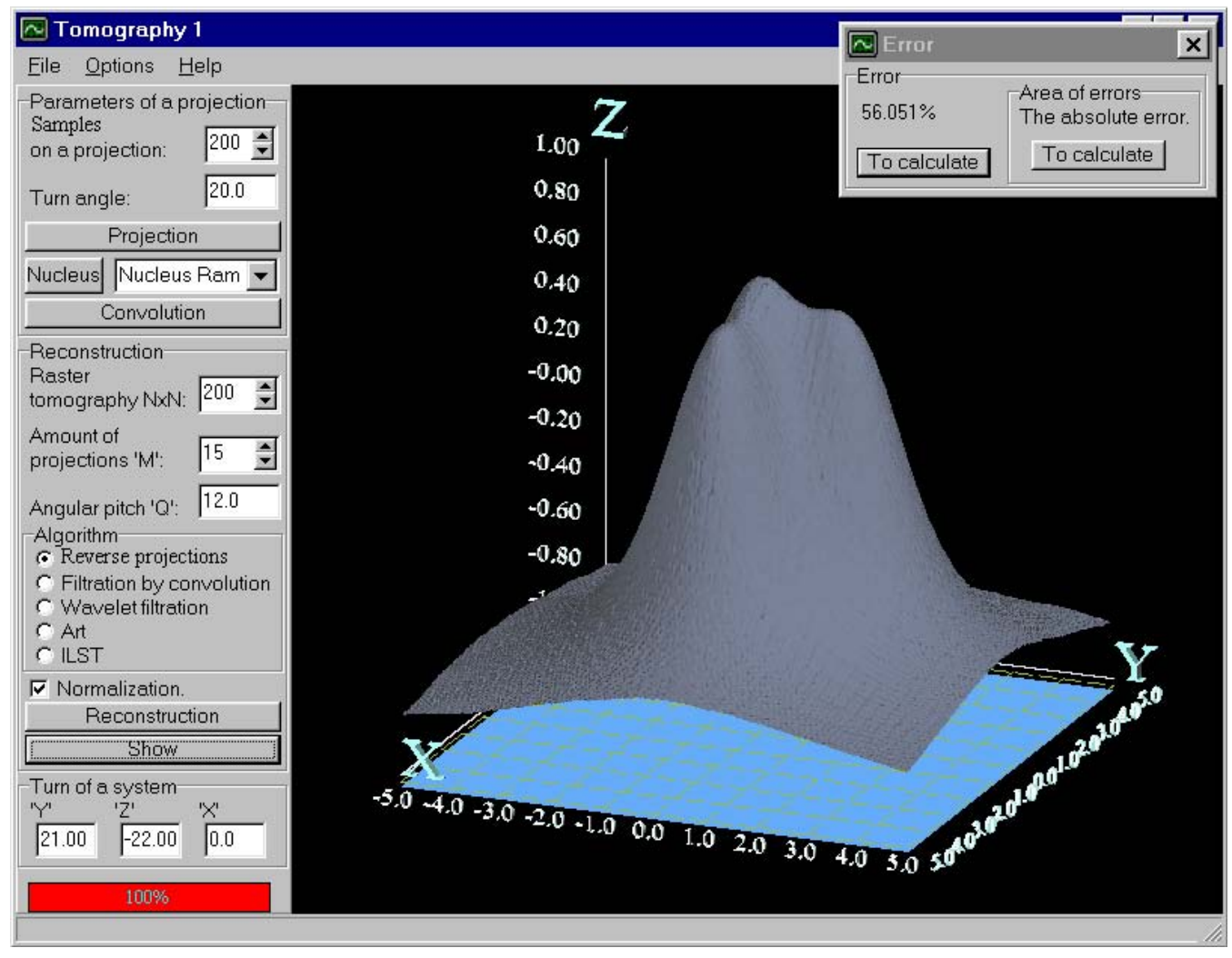

Fig. 2.14. A method of return projections (error $55.051 \%$ ). 


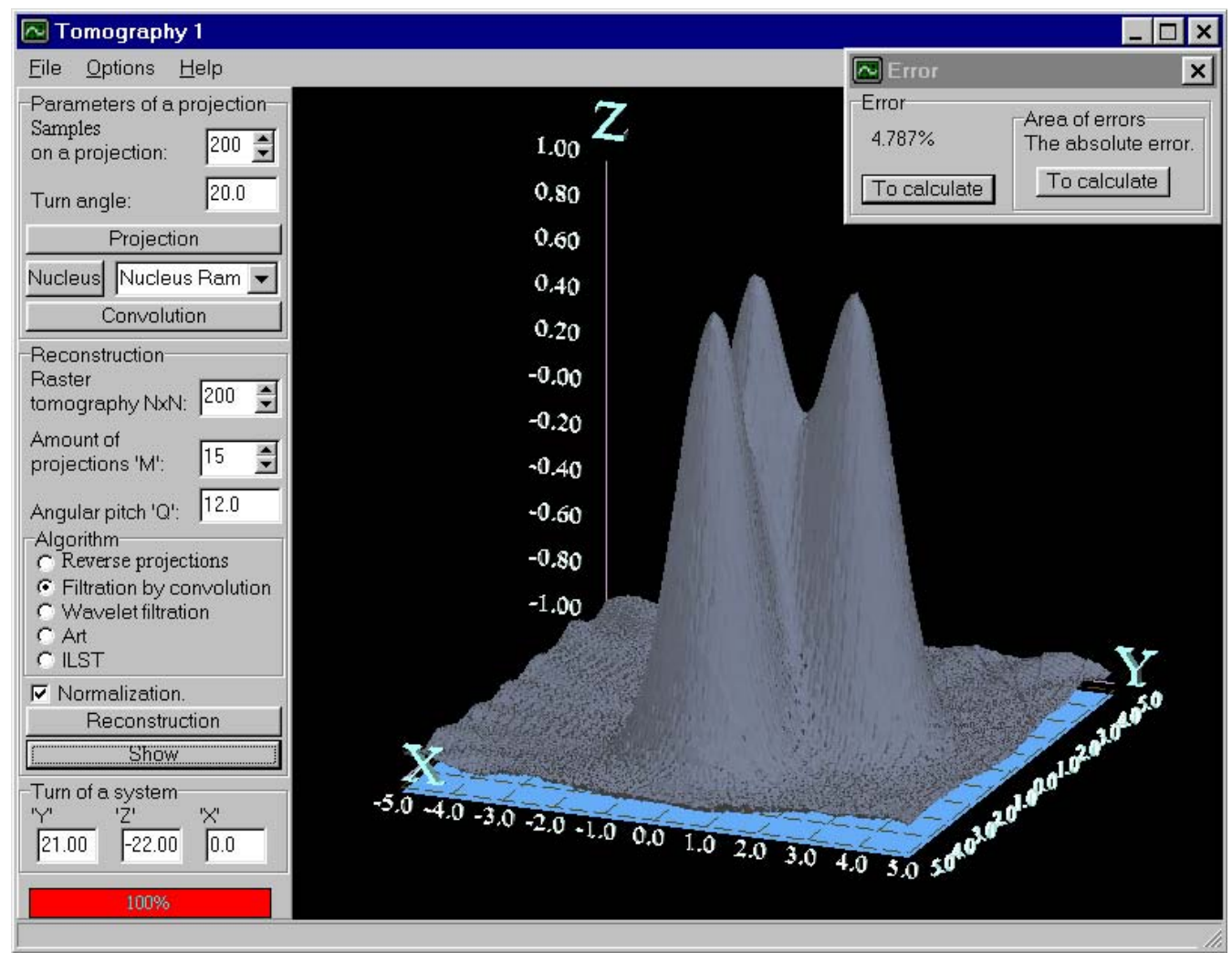

Fig. 2.15. A method of a return projection with a filtration

by convolution (error $4.787 \%$, nucleus Ramachandran).

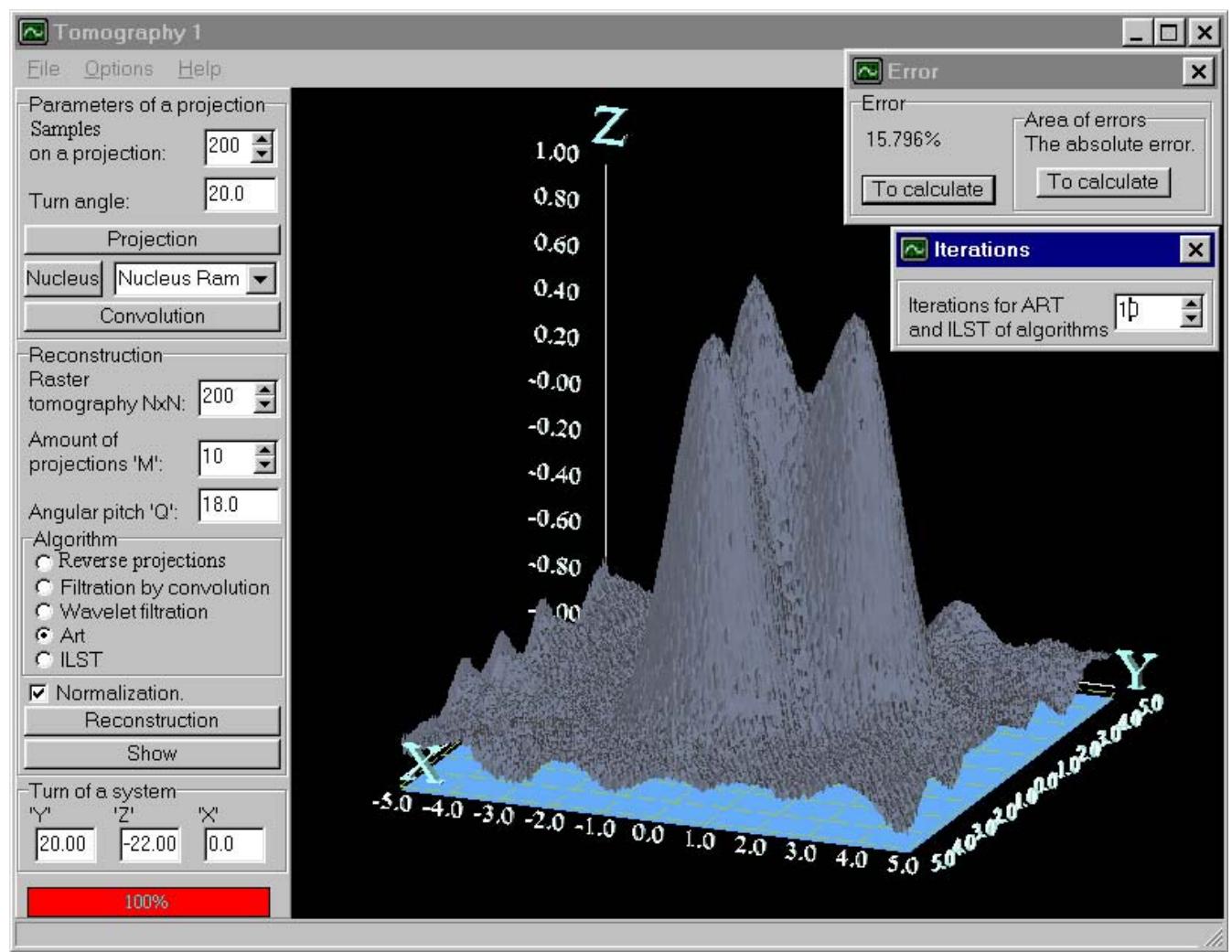

Fig. 2.16. A method ART (error $15.796 \%, 10$ iterations). 


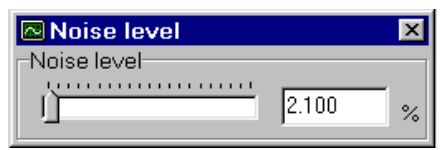

区

Fig. 2.18. Superposition of noise.

Student is offered to conduct a series of experiments, imposing on a projection noise, to make the comparative qualitative and quantitative analysis of obtained outcomes. Below for an example the outcomes of restoring from 2 $\%$ by a noise are indicated.

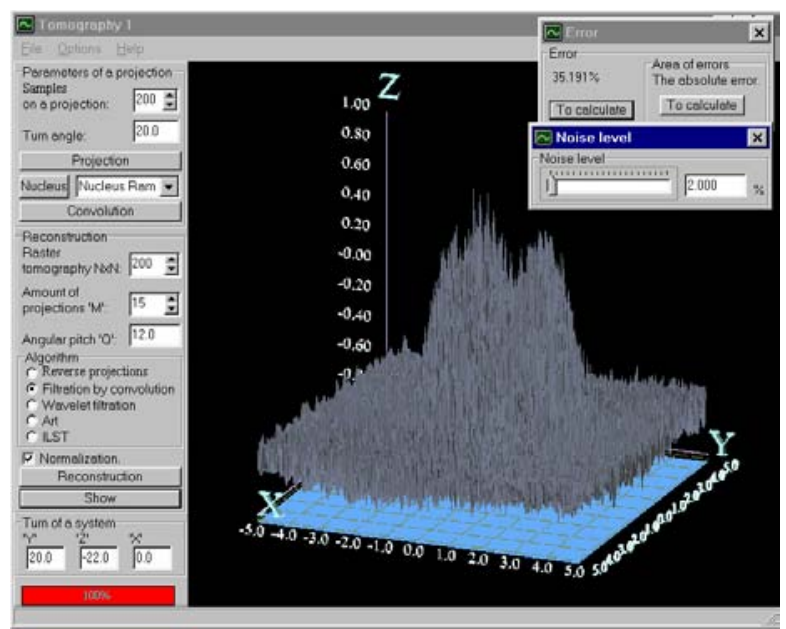

Fig. 2.17. A method of a revers projection with a filtration by a convolution (12 projections, error $35.191 \%$, noise $2 \%$, nucleus Ramachandran, for a comparison see. Fig. 2.15).

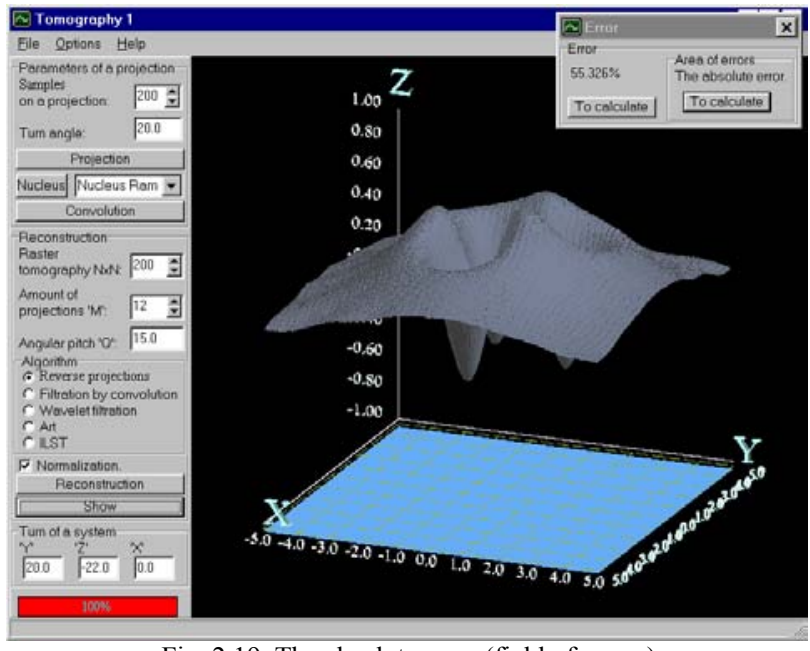

Fig. 2.19. The absolute error (field of errors).

Residual between an ideal model and restored by a method of a return projection.

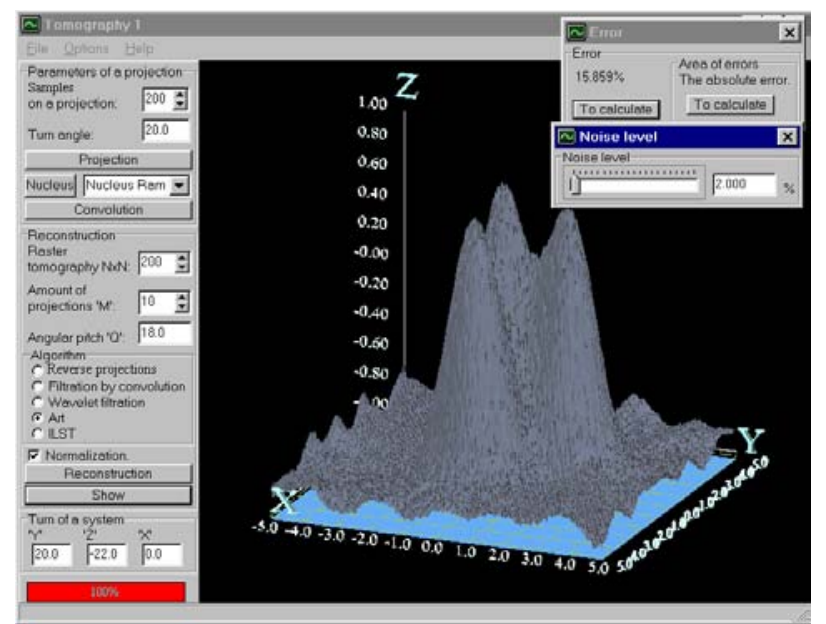

Fig. 2.18. A method ART (10 projections, error $15.859 \%$, noise $2 \%$, for a comparison see Fig. 2.16.).

Besides the information about a general error of restoring, user is grantiven a possibility of evaluating visually the absolute error of each restored object element of object «The absolute error» (Fig. 2.13.).

The absolute error is removed as the certain field of errors (Fig. 2.19.).

\begin{tabular}{|c|c|c|}
\hline \multirow{2}{*}{\multicolumn{3}{|c|}{$\begin{array}{l}\text { Set-up of an image } \\
\text { Set-up of an image }\end{array}$}} \\
\hline & & \\
\hline$\Gamma$ Points: & V Site & $\sqrt{v}$ Grid \\
\hline 『 Triangulation & V Axes & $Г$ Halftints \\
\hline
\end{tabular}

Fig. 2.20. Parameters of map.
The possibility of maximum visualizing the outcomes of numerical experiment is grantiven to student, who is changing parameters of restored object map (Menu «Options», Set-up of an image ...) (Fig. 2.20.) «Points» to map object by points (Fig. 2.21.) (thus, the computing resources are essentially saved); «Triangulation» - triangulation of object; «Halftints» - to map object by halftints (Fig. 2.22.); «Site» - to map a grid; «Axes» - to show axeses of coordinates; «Grid» - to show the basis. 


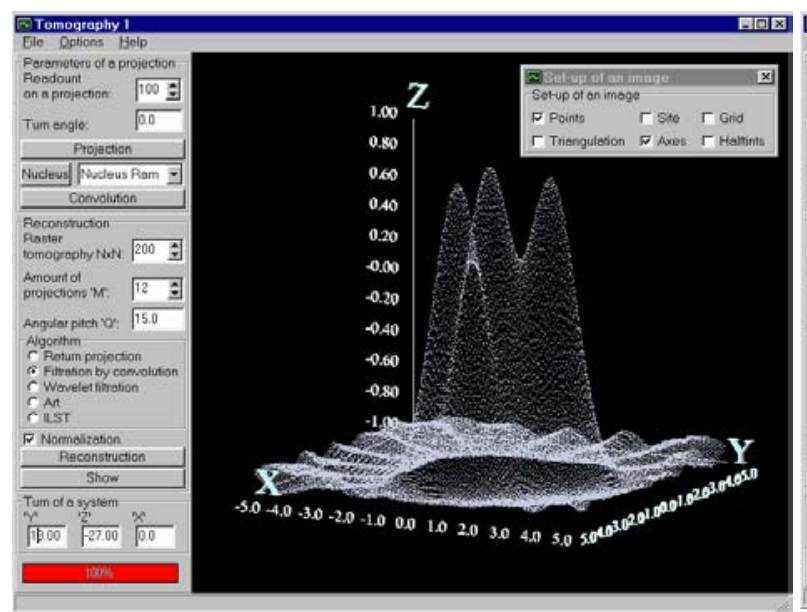

Fig. 2.21. Display of object by points

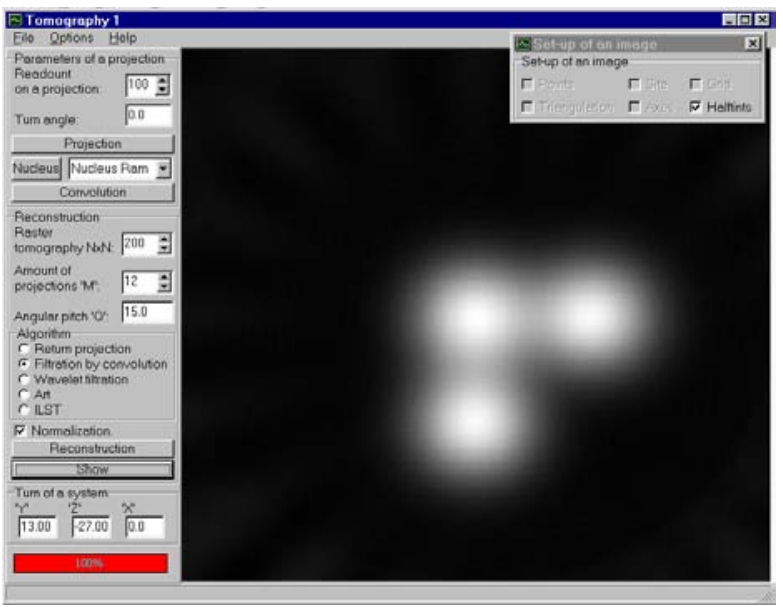

Fig. 2.22. Display of object by halftints.

Now program is successfully used for training students in program «Optical methods of plasma diagnostics ».

\section{References}

1. V.V. Pikalov and T.S. Melnikova. Tomography of Plasma ( Novosibirsk, Science,1995)

2. Sinkov M.V., Ternovoy K.S, "Introduction in modern tomography", (Kiev, the Scientific idea, 1983), p. 281.

3. Pikalov V.V., Preobrazhenskiy N.G., "Computing tomography and physical experiment", Physics-Uspehi, 143, pp. $469-499$ (1983)

4. Denisova N.V., "Fan tomography of gas and plasma because method maxima of an entropy", Optics and spectroscopy, 83, pp. 1019-1024

(1997)

5. M.M. Astaf'eva, "Wavelet analysis: basic theory and some application", Physics-Uspehi , 166, pp. 1145-1170 (1996) 